\title{
Reduction Mammoplasties in Adolescents: Examining Motivations and Resources Available to Patients
}

\author{
Caroline $\mathrm{J}_{0}{ }^{1}$, Stacey Gil ${ }^{1}$ and Virginia Clark ${ }^{1}$ \\ Ramsey High School, Ramsey, NJ, USA \\ ABSTRACT
}

Women who have abnormally large breasts often choose to undergo reduction mammoplasty, also known as breast reduction surgery. There have been many studies examining the psychological and medical reasons underlying why adult women seek such an operation as well as its postoperative effects. However, adolescents with enlarged breasts lack the knowledge and institutional support for their operations. This paper will examine the existing literature on adolescent reduction mammoplasties and comment on the lack of infrastructure and support for patients and their families.

\section{Cosmetic Breast Surgery in Adult Women}

Of all cosmetic surgical procedures, one of the most prominent and most influential in women's lives is breast surgery (Crerand \& Magee, 2013). Breast augmentations and breast lifts are currently the most popular forms of cosmetic procedure, with more than 400,000 operations performed in 2019. These procedures are purely cosmetic, and patients are therefore not given institutional support or insurance coverage (American Society of Plastic Surgeons, 2019; The Aesthetic Society, 2019). However, the next most popular operation, breast reduction surgery, also known as reduction mammoplasty, exhibits qualities of both an aesthetic and a medical procedure. Many women with larger breasts, a medical condition known as macromastia, experience a complex mixture of social and physical social pressures that influence their decision to opt in for such a drastic surgery (Healthwise, 2020). There have been many studies examining the reasons that adult women seek mammoplasty and its postoperative effects. (Reardon \& Grogan, 2010; Crerand \& Magee, 2009; Horch et al., 2009). However, these life changing operations are often overlooked within the younger population. This paper will examine the existing literature on adolescent cosmetic surgery and propose solutions to provide support for adolescent patients who are suffering from a low quality of life due to their breasts.

\section{Motivations for Undergoing Reduction Mammoplasty}

The popularity of cosmetic surgery can be attributed to several different factors. First, cosmetic medical procedures have significantly improved and become safer for patients. Furthermore, the acceptance of cosmetic surgery, aided by the prevalence of advertisements, has significantly increased amongst women, with 55\% approving cosmetic surgeries and 30\% stating that they would like to have such an operation in the future (Cash, 1996).

The reasons for choosing to undergo reduction mammoplasties can be largely divided into two categories: physical and psychological. The primary motivation for women seeking reduction breast surgery is the physical pain and discomfort due to the disproportionate size of their breasts. Back pain, cramps, and poor postures are common symptoms that pose a significant disruption in daily life (Reardon \& Grogan, 2010). Within this cohort, women report generally improved quality of life post-operation (Horch, 1999).

Additionally, women who seek cosmetic breast surgery were found to have a variety of psychological symptoms and conditions. The negative self body image stemmed from three main factors: perceptual, sociocultural and 
developmental (Cash, 1996). As to perceptual factors, women describe the physical attributes that are not in line with the objective reality of their bodies, often describing their features as "ugly" and "grotesque" (Cash, 1996; Crerand \& Magee, 2009). Sociocultural factors also have a significant effect on women's psyche. The emotional pain and insecurity that girls feel in their adolescent years persist and influence their decision to seek cosmetic surgery as adults. Developmental factors denote the physical maturation of girls throughout their adolescent years. Many feel that they receive unwanted attention during their developmental years and wish to have their proportions match those of their peers (Cash, 1996; Grogan \& Reardon, 2010).

Although it has been shown that most(?) women are satisfied with their operations, there are conflicting results about the definitive psychological benefits of cosmetic surgery. A portion of patients who receive cosmetic medical procedures shows evidence of exhibiting psychological symptoms due to their poor mental health (Cash, 1996). There is also a risk of women failing to feel satisfied with their procedure and continuing to seek other cosmetic surgeries to alter their physical appearance. Empirical studies have demonstrated that such patients often display symptoms of eating disorders and suicide (Grogan \& Reardon, 2010). The vulnerabilities of patients will only be compounded in adolescence, as they are still developmentally immature and heavily influenced by social factors.

\section{Social Conditions and Physical Development During Adolescence}

As adult women report that their negative body image substantially influences their decision to undergo breast surgery, there needs to be an acute understanding of the social environments in which adolescents develop. As adolescents develop self-esteem and self-concept, body image (or perceptions and attitudes regarding their own physical appearance) is one of the largest factors in their mental and emotional wellbeing (Crerand \& Magee, 2013). Adolescent boys and girls are affected differently as they go through puberty. While the physical attributes of boys become more desirable as they grow taller, faster, stronger, and more muscular, girls develop both "desirable" and "undesirable" traits. Their secondary sexual characteristics become more developed, but fat percentage significantly increases (Stang et al., 2005). As a result, puberty tends to have detrimental effects upon an adolescent girl's self-perception, far more so than for adolescent boys. In fact, studies demonstrate that 50-88\% of adolescent girls feel negatively about the shape or size of their bodies (Stang et al., 2005). Adolescent girls are especially sensitive and aware of their own breast development (needs citation), and enlarged breasts can contribute to an adolescent girl's negative perception of her body. Some who suffer from macromastia will particularly be self-conscious about their bodies undergoing a dramatic change. Starting from their teenage years, adolescent girls undergo dramatic transitions, or puberty, that affect their body and appearance (Voelker, et al., 2015). Preliminary studies have shown that older adolescents going to college are dissatisfied with their body image as well, with $77 \%$ dissatisfied with their breasts specifically (Ganesan et al., 2018).

Modern media further exacerbate the stigma surrounding body image, particularly in adolescents (Stang et al., 2005). A study demonstrates that, should Barbie, originally a doll that later evolved into the beauty standard for impressionable young girls around the world, existed in real life, she would have a 22-inch waist and 40-inch chest, compared to the 28-inch waist and 35-inch chest of the average female (Brownell \& Napolitano, 2006). Adolescent girls exposed to Barbie and other stereotypical beauty standards in media, such as the average 5'10, 110-lb model, begin to harbor unrealistic expectations for their bodies, and, when they inevitably fall short in reality, they may develop body image issues or eating disorders (Stang et al., 2005).

Cosmetic breast surgery, therefore, is an understandably controversial subject when applied to adolescent girls. It has been recommended that adolescent girls seeking cosmetic breast surgery undergo significant psychological evaluation before undergoing surgery (Crerand \& Magee, 2013). 


\section{Cosmetic Surgery in Adolescents}

Although cosmetic surgery amongst women has been extensively studied, there is still not much information about cosmetic surgery within the adolescent population. If there are risks among the adult female population, there is a significant risk of adolescents exhibiting similar psychological and physical symptoms (Crerand \& Magee, 2013).

Despite the paucity of research, there have been some studies revealing that reduction mammoplasties for adolescents lead to an improved quality of life post-operation. Within young girls who have macromastia or general overdevelopment of breasts, most have a lower quality of life and subsequent eating disorders regardless of BMI index. Similar to adult women, adolescent girls suffer from physical and mental health issues. Adolescents with macromastia exhibit breast, neck, back, and shoulder pain along with difficulties finding clothes and playing school sports. In addition, this cohort also experiences a higher incidence of depression and anxiety (Cerrato et al., 2012). Studies have also concluded that there is a difference in outcomes for girls of different races, with Hispanics and the African American population showing faster development than their White counterparts (Cerrato et al., 2012; Biro et al., 2010).

Nearly $80 \%$ of girls who received reduction mammoplasties report significant improvements in their quality of life and physical relief of symptoms from pre-operation. Furthermore, more than $90 \%$ of girls would recommend the procedure to a friend suffering from similar symptoms (McMahan et al., 1995). Although the psychological and physical effects of cosmetic breast surgery have been analyzed within girls who undergo surgery, there is a severe lack of research on the perception of these procedures on adolescents among their peers. Adolescence is a turbulent period in which puberty and the social environment shapes opinions significantly, so relevant research would shed critical light on how peers can impact a female adolescent's decision to opt in for a possibly beneficial surgery.

Considering the scale of body image issues in adolescents, particularly in girls, reduction mammoplasties may be as viable an option for adolescents as it is for adults (Crerand \& Magee, 2013). Though the evidence is not entirely conclusive, it points towards the psychological soundness of adolescent patients for cosmetic surgery and the potential postoperative psychological benefits of undergoing such a procedure (Crerand \& Magee, 2013).

\section{Resources Available for Women}

Currently there is little information available for any institutional support for adolescent girls who have enlarged breasts or suffer from macromastia. Most information on resources available to patients mostly focus on adult women. In this section, we will examine the current resources that are offered to women who seek breast reduction surgery.

\section{Insurance}

Any surgery considered medically reconstructive is both indicated and covered by health insurance. In contrast, if a surgery is performed with cosmetic or aesthetic considerations, patients are forced to pay for the operation out of pocket. For reduction mammoplasty patients, the standards for medical consideration are quite high. The surgery is defaulted as a cosmetic procedure and often paid for by the patient out of pocket. If the patient is able to provide "adequate" proof that the surgery is medically necessary, an insurance company may consider covering the procedure (Chidyllo, 2018). However, the act of citing "adequate" proof demonstrating necessity for the procedure is cumbersome, prolonged, and costly. 
Table 1. Requirements for Insurance Coverage for Reduction Mammoplasty based on Aetna and United Healthcare Insurance Coverage for Reduction Mammoplasty

1. Patient shows ongoing symptoms of at least 2 of the body areas below, directly due to macromastia and affects daily activities for at least 1 year:

1. Headaches

2. Shoulder, upper back, neck, pain

3. Painful kyphosis

4. Pain/discomfort/ulceration from bra straps cutting into shoulders

5. Skin breakdown overlying breast tissue

6. Upper extremity paresthesias

2. Provide photographic documentation of breasts and the doctor determines that there is definite proof that:

a. There is a reasonable likelihood that the member's symptoms are primarily due to macromastia; and

b. Reduction mammoplasty is likely to reduce the severity of chronic pain; and c. Pain symptoms continue despite being prescribed therapeutic measures for three months

3. Medical professionals need to remove breast tissue based on the surface area of the breast. There will be restrictions on how much can be removed based on the Mosteller formula

In most cases, insurance companies require 2-3 documented reports from referred specialists before even considering covering the procedure for practical reasons. Following this prerequisite, insurance companies often request 6-12 months of documentation and treatment by a physical therapist, chiropractor, dermatologist, or orthopedist citing macromastia as the source of various debilitating symptoms (Chidyllo, 2018). The entire process takes around 3-6 months, which, for women who suffer from macromastia and seek breast reduction, is another 3-6 months of physical torment.

For women who seek breast reduction to resolve the aforementioned psychological issues with body-perception, insurance coverage is a lengthy and complicated process (Behring \& Biggers, 2020). The Paul Wellstone and Pete Domenici Mental Health Parity and Addiction Equity Act (also known as the mental health parity law or federal parity law) requires mental health, behavioral health, and substance-abuse health to be covered by health services on the same scale as physical health. Unfortunately, the law is widely underutilized: a 2014 APA study found that more than 90 percent of Americans were unfamiliar with the mental health parity law. The act does not require that mental health is insured under the health service; it merely states that, if mental health benefits are offered, they cannot have more restrictive requirements than those that apply to physical health benefits. Still, most large insurance companies had already offered mental health service prior to the law's ratification (American Psychological Association, 2014).

Despite the federal parity law and other comparable measures, insurance companies continue to take advantage of clients who do adequately demonstrate psychological necessity for a medical treatment. A class action lawsuit brought in California questioned the validity of the restrictive criteria that must be met to prove medical necessity and guarantee insurance coverage for mental health treatment. In Wit v, United Behavioral Health, individuals sued a plan benefits administrator for denial of care for outpatient or residential treatment for mental health or substance use based on the plan members' failure to meet criteria in level of care or coverage determination guidelines. The court determined that United Behavioral Health's denial of benefits was "arbitrary and capricious," and that its coverage guidelines were influenced by financial interests (Bogusz, 2020). 
The United Behavioral Health is certainly not alone in its controversy with the gray area of insurance coverage of mental health treatment. Although mental health is increasingly recognized as a significant aspect of an individual's general health, the overall lack of insurance coverage of psychological treatments remains a barrier to be overcome, especially in the context of reduction mammoplasties, where the line between physical and mental necessity requires further clarification.

Although cosmetic procedure is perhaps the most obvious avenue through which an adolescent may resolve debilitating psychological effects of macromastia, other unofficial, less-invasive options exist to combat body-image issues, the foremost of which is community. Community provides key advantages to an individual suffering with psychologically and socially debilitating symptoms: a sense of belonging, support, and purpose. Through belonging, individuals learn that they are not alone in their respective struggles with body image perception. Further, a community providing support to an individual allows them to feel cared for and safe, which can ultimately make an impactful difference on a person's outlook on life. Finally, being part of a community gives an individual a sense of purpose and motivation to help others struggling with similar issues (GirlTalkHQ, 2014).

\section{Unofficial Online Forums for Breast Reduction}

One common sentiment among post-operative reduction mammaplasty patients is that they wish they had opted for the procedure far earlier, perhaps even in adolescence. However, the resources available to women with concerns about the size or shape of their breasts are limited. For adolescent girls in comparable situations, the already-inadequate variety of resources wanes significantly. RealSelf, an organization dedicated to helping people learn more about cosmetic procedures, share their experiences, and connect with top experts in the field of cosmetic treatments, is a leading company that aims to help women make more informed decisions. Through an unprecedented access to before and after photos, "Worth It" ratings, and personalized answers from aesthetic experts, the response to RealSelf has been overwhelmingly positive, with one user even exclaiming, "I consider the RealSelf community my family that gives me important advice and tips, to make my post-op experience a little less uncomfortable." A website, directed specifically to the unique needs accompanying adolescent struggles with breasts could potentially reap unprecedented benefits for young girls as well as reduce the number of women who regret waiting so long to undergo a breast reduction. (RealSelf)

As it stands, no online forums or communities exist to discuss adolescent girls' specific struggles with macromastia and experiences with reduction mammoplasties. However, in Australia, a Sydney city council team is tackling harmful social media trends that exacerbate self-confidence issues by creating a forum dedicated to helping teen girls and parents reach a balance when it comes to body image and the media they consume (Gilbert, 2019). Further, the National Eating Disorders Association promotes healthy attitude towards body image through an online forum where adolescents can form meaningful connections by discussing self esteem issues and recovery for eating disorders, which can arise in individuals struggling with macromastia (National Eating Disorders Association, 2018; Losee et al., 1997).

\section{Medical and Social Support: a Two-Pronged Solution.}

Official treatment for macromastia includes weight management, physical therapy and, of course, reduction mammoplasty. Adolescents concerned with the size or shape of their breasts may contact a reputable plastic surgeon to schedule a consultation and discuss their concerns as well as determine whether a reduction mammoplasty is both possible and advisable considering their respective cases (Snodgrass, 2012). However, the guidelines for young girls who wish to have financial support for the procedure is unclear. For the mental and physical wellbeing of many young women, there needs to be clearer guidelines and options for adolescents who seek breast surgery. 
While reduction mammoplasty is the most direct manner through which to resolve breast hypertrophy, it may not be within the budget or other limitations of the individual adolescent. A far more approachable and accessible solution may lie in the aforementioned discussion of the significance of community and understanding. An adolescent struggling with abnormal or perceived abnormal breast size or shape may seek local communities or forums in which they discuss their experiences with macromastia and find solace in the community. Existing forums, such as RealSelf, can offer guidance to young women through a moderated and separate section. Through these communities of like-minded individuals with uniquely-shared experiences, adolescents can discover that they are not alone in their struggles and work with one another to rehabilitate their self-confidence and body perception.

\section{Acknowledgments}

I would like to thank my advisors Stacey Gil and Virginia Clark for helping me with this project.

\section{References}

American Psychological Association. (2014, May 14). Does your insurance cover mental health services? American Psychological Association. https://www.apa.org/topics/parity-guide.

American Society of Plastic Surgeons. (2019). 2019 Plastic Surgery Statistics Report (pp. 1-25).

Behring, S. (2020, June 30). Does Medicare Cover Breast Reduction Surgery? Healthline. https://www.healthline.com/health/medicare/does-medicare-cover-breast-reduction\#medicare-coverage.

Biro, F. M., Galvez, M. P., Greenspan, L. C., Succop, P. A., Vangeepuram, N., Pinney, S. M., Teitelbaum, S., Windham, G.C., Kushi, L.H., \& Wolff, M. S. (2010, September 1). Pubertal Assessment Method and Baseline Characteristics in a Mixed Longitudinal Study of Girls. American Academy of Pediatrics. https://doi.org/10.1542/peds.20093079

Breast Reduction Surgery and Gynecomastia Surgery - Medical Clinical Policy Bulletins | Aetna. (n.d.). Health Insurance Plans; Aetna. http://www.aetna.com/cpb/medical/data/1_99/0017.html

Brownell, K. D., \& Napolitano, M. A. (2006, February 13). Distorting reality for children: Body size proportions of Barbie and Ken dolls. Wiley Online Library. https://doi.org/10.1002/1098-108X(199511)18:3<295::AID-

EAT2260180313>3.0.CO;2-R

Body Image Forum Analyzes Social Media Impact On Teen Girls. GirlTalkHQ. (2014, July 25). https://www.girltalkhq.com/body-image-forum-analyzes-social-media-impact-on-teen-girls/.

Bogusz, G. B. (2020, March 13). Health Insurers Still Don't Adequately Cover Mental Health Treatment. National Alliance of Mental Illness. https://www.nami.org/Blogs/NAMI-Blog/March-2020/Health-Insurers-Still-Don-tAdequately-Cover-Mental-Health-Treatment.

Cash, T. F. (1996, December 1). Body Image and Cosmetic Surgery: The Psychology of Physical Appearance. SAGE Journals. https://doi.org/10.1177/074880689601300407 
Cerrato, F., Webb, M. L., Rosen, H., Nuzzi, L., McCarty, E. R., DiVasta, A. D., Greene, A.K., Labow, B. I. (2012, August 1). The Impact of Macromastia on Adolescents: A Cross-Sectional Study. American Academy of Pediatrics. https://doi.org/10.1542/peds.2011-3869

Chidyllo, S. (2018, January 19). Is breast reduction covered by health insurance? American Society of Plastic Surgeons. https://www.plasticsurgery.org/news/blog/is-breast-reduction-covered-by-health-insurance.

Crerand, C. E.; Infield, A. L. BA; Sarwer, D. B. (2009, March). Psychological Considerations in Cosmetic Breast augmentation. Official Journal of the International Society of Plastic and Aesthetic Nurses 29(1):49-5. DOI: 10.1097/01.PSN.0000347725.13404.5f

Crerand, C. E., \& Magee, L. (2013, February). Cosmetic and reconstructive breast surgery in adolescents: psychological, ethical, and legal considerations. Seminars in plastic surgery. DOI: 10.1055/s-0033-1343999

Community. National Eating Disorders Association. (2018, February 22). https://www.nationaleatingdisorders.org/community.

Ganesan, S., Ravishankar, S. L., \& Ramalingam, S. (2018, December). Are Body Image Issues Affecting Our Adolescents? A Cross-sectional Study among College Going Adolescent Girls. Indian journal of community medicine: official publication of Indian Association of Preventive \& Social Medicine. DOI: 10.4103/ijcm.IJCM_62_18

Gilbert, S. (2019, November 18). The Importance of Community and Mental Health. National Alliance of Mental Illness. https://www.nami.org/Blogs/NAMI-Blog/November-2019/The-Importance-of-Community-and-MentalHealth.

Healthwise. (2020, July 02). Breast Reduction. Retrieved from https:/www.cigna.com/individuals-families/healthwellness/hw/medical-topics/breast-reduction-tf2235.

Horch, R. E., Jaeger, K., \& Stark, G. B. Quality of Life After Breast Reduction: Annals of Plastic Surgery. LWW. (1999). DOI: 10.1097/01.sap.0000171444.79737.70

Losee, J. E., Serletti, J. M., Kreipe, R. E., \& Caldwell, E. H. (1997). Reduction mammaplasty in patients with bulimia nervosa. Annals of plastic surgery. DOI: 10.1097/00000637-199711000-00001

McMahan, J. D., Wolfe, J. A., Cromer, B. A., \& Ruberg, R. L. (1995, September). Lasting success in teenage reduction mammaplasty. LWW. DOI: 10.1097/00000637-199509000-00001

Reardon, R., Grogan, S. (2010, July 23). Women's Reasons for Seeking Breast Reduction: A Qualitative Investigation. Journal of Health Psychology, 16(1), 31-41. https://doi.org/10.1177/1359105310367531

Reel, J., Voelker, D., \& Greenleaf, C. (2015). Weight status and body image perceptions in adolescents: current perspectives. Adolescent Health, Medicine and Therapeutics, 149. https://doi.org/10.2147/ahmt.s68344 
Snodgrass, B. (2012, November 9). Large breasts causing back pain? Breast reduction surgery offers relief. American Society of Plastic Surgeons. https://www.plasticsurgery.org/news/blog/large-breasts-causing-back-pain-breastreduction-surgery-offers-relief.

Stang, J., Story, M. T., Croll, J. (2005). Chapter 13: Body Image and Adolescents. In Guidelines for adolescent nutrition services (pp. 155-166)., Center for Leadership, Education and Training in Maternal and Child Nutrition. http://citeseerx.ist.psu.edu/viewdoc/download;jsessionid=2A1F82E0F6297A6AABC04D90601624B1?doi=10.1.1.620.934\&rep=rep1\&type=pdf

The Aesthetic Society. (2019). Aesthetic Plastic Surgery National Databank Statistics (pp. 1-26). 